\title{
Selective Adsorption and Efficient Removal of Phosphate from Aqueous Medium with Graphene-Lanthanum Composite
}

Mingli Chen, Chunbao Huo, Yikun Li, Jianhua Wang*

Research Center for Analytical Sciences, College of Sciences, Northeastern University, Box 332, Shenyang 110819, China

*Corresponding author.

E-mail: jianhuajrz@mail.neu.edu.cn (J.-H Wang).

Tel: +86 24 83688944; Fax: +862483676698 


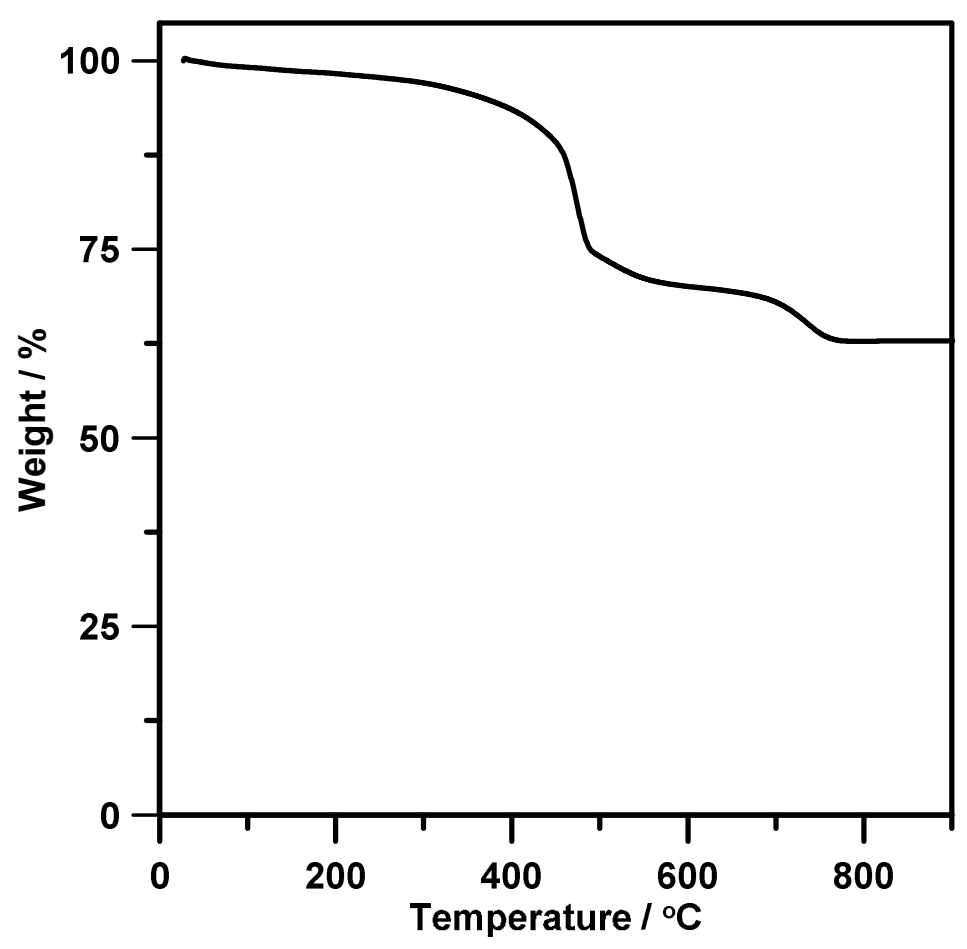

Fig. S1. TGA curves of the lanthanum oxide decorated graphene 


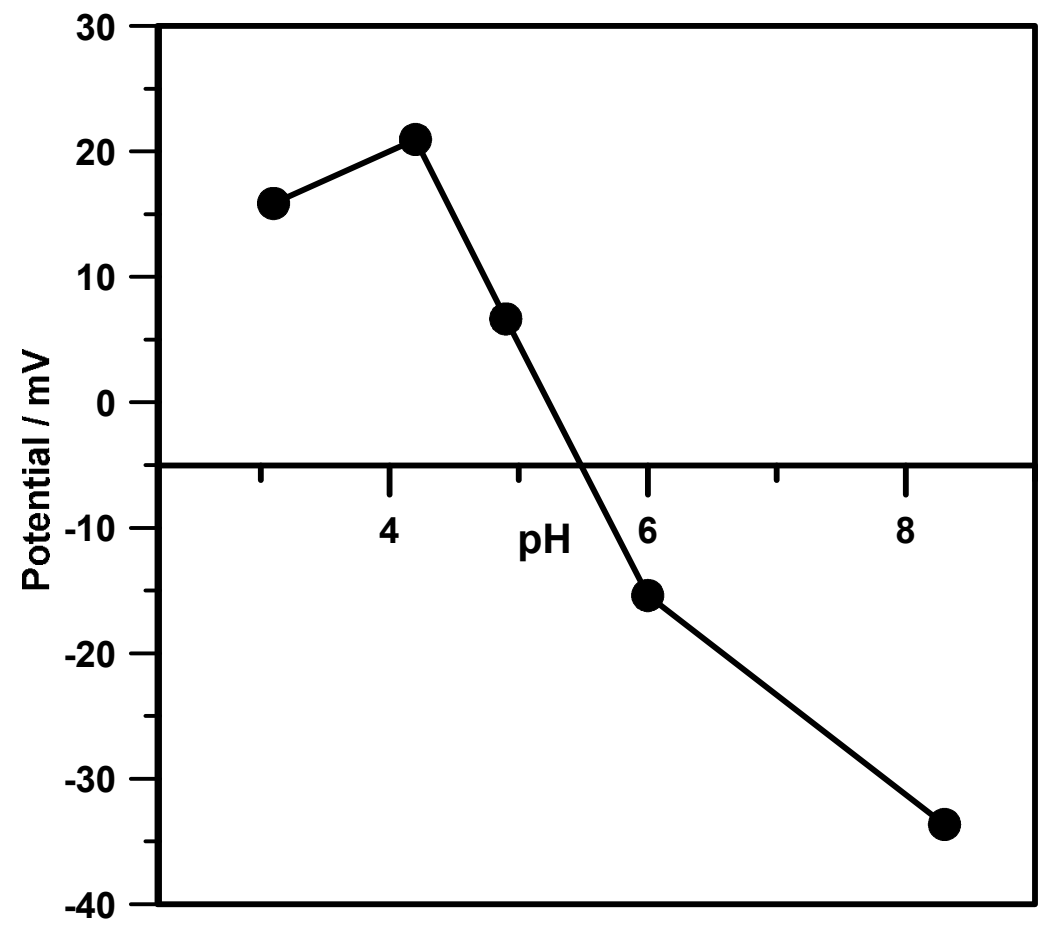

Fig. S2. Surface charge property of lanthanum oxide decorated graphene 


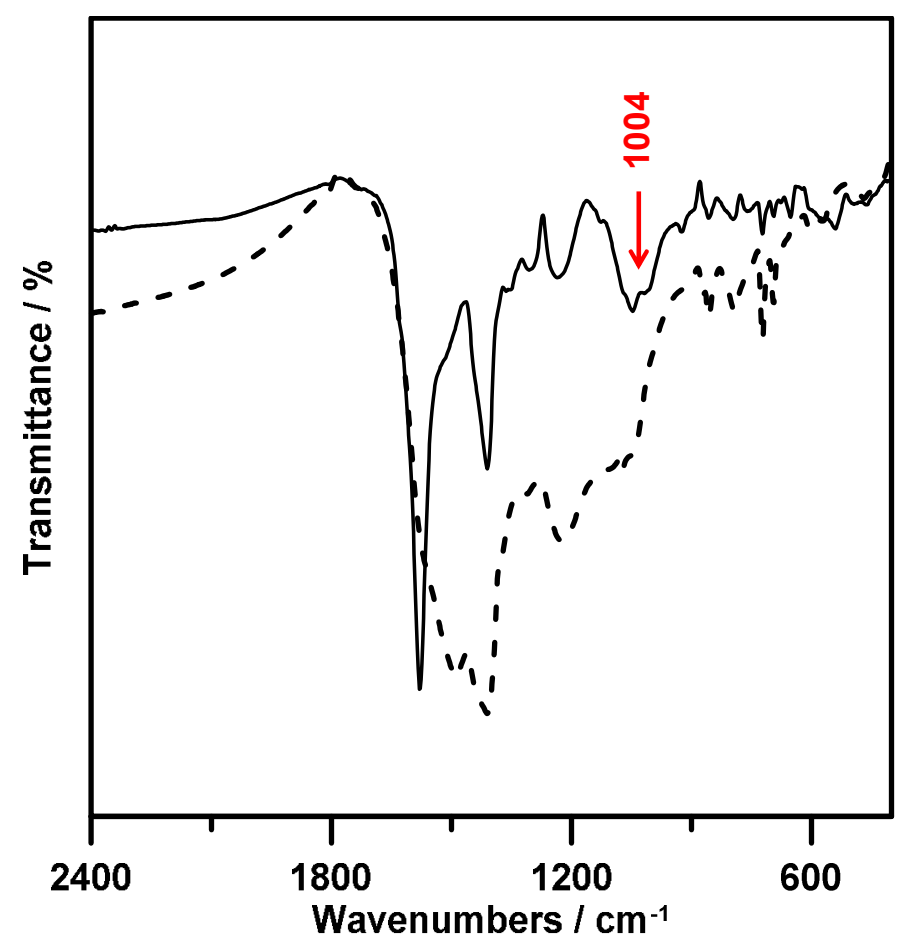

Fig. S3. FT-IR Spectra for the 3D graphene- $\mathrm{La}_{2} \mathrm{O}_{3}$ composites (dashed line) and for that after adsorption of phosphate at $142 \mathrm{mg} \mathrm{L}^{-1}$ (solid line). 